\title{
TWO DIMENSIONAL QUANTUM GRAVITY AND RANDOM SURFACES
}




\section{This page is intentionally left blank}


JERUSALEM WINTER SCHOOL FOR THEORETICAL PHYSICS
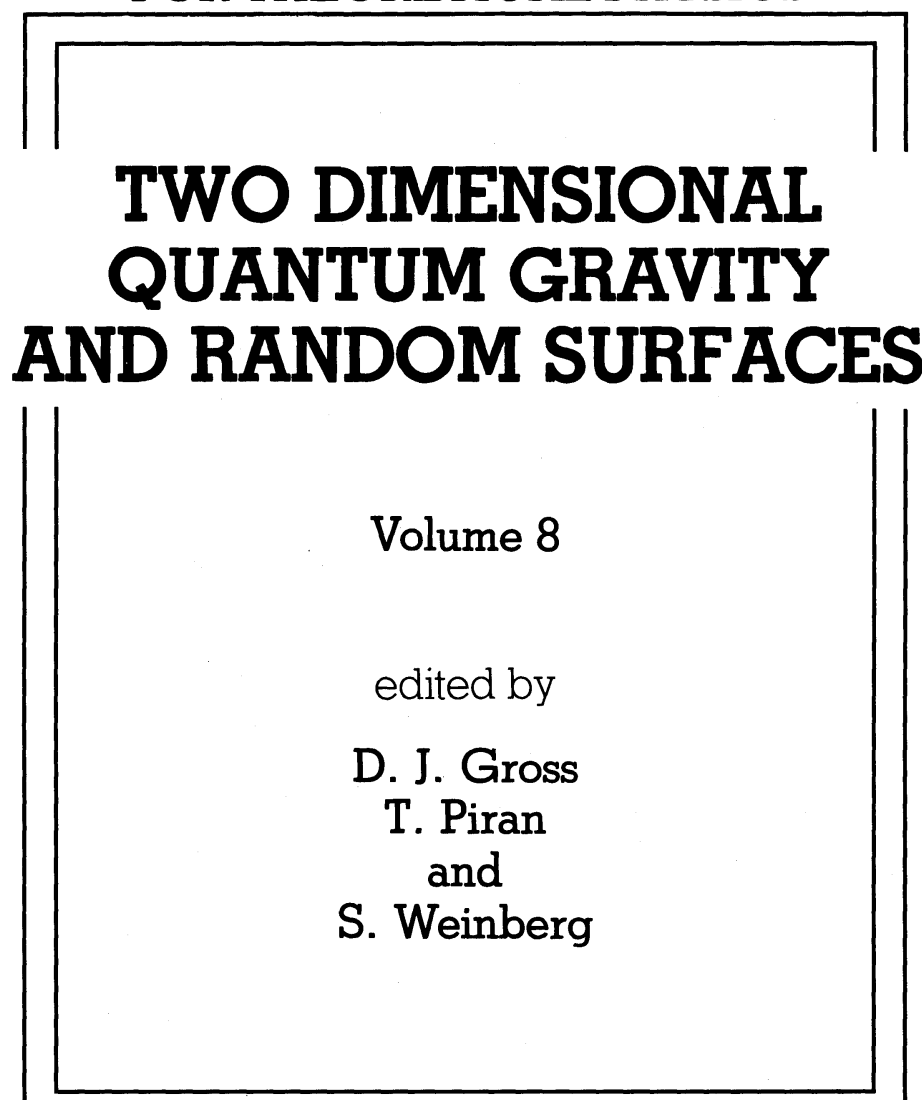

Jerusalem, Israel

27 Dec 90 - 4 Jan 91

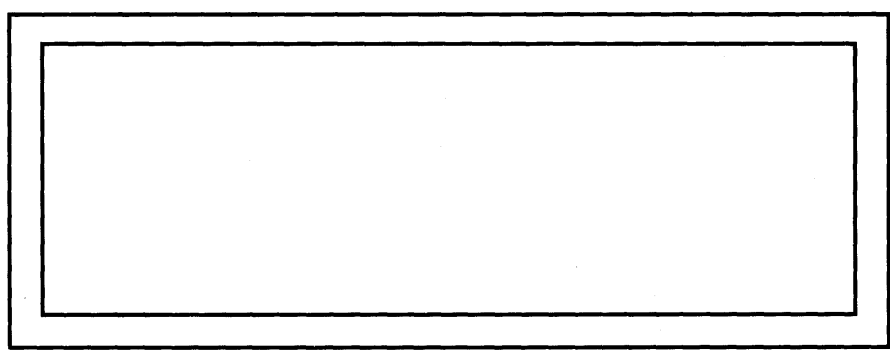




\section{Published by}

World Scientific Publishing Co. Pte. Ltd.

P O Box 128, Farrer Road, Singapore 9128

USA office: Suite 1B, 1060 Main Street, River Edge, NJ 07661

UK office: 73 Lynton Mead, Totteridge, London N20 8DH

\section{TWO DIMENSIONAL QUANTUM GRAVITY AND RANDOM SURFACES}

Copyright @ 1992 by World Scientific Publishing Co. Pte. Ltd.

All rights reserved. This book, or parts thereof, may not be reproduced in any form or by any means, electronic or mechanical, including photocopying, recording or any information storage and retrieval system now known or to be invented, without written permission from the Publisher.

ISBN 981-02-0642-9

ISBN 981-02-0643-7 (pbk)

Printed in Singapore by JBW Printers and Binders Pte. Ltd. 


\section{PREFACE}

The Eighth Jerusalem Winter School on Two Dimensional Quantum Gravity and Random Surfaces was held from December 27, 1990 to January 4, 1991. In the past few years there has been much study of two dimensional quantum gravity or random surfaces. These provide simple models of string theory as well as toy models of quantum gravity. They also have possible applications to the Statistical Mechanics of phase boundaries and to the development of an effective string description of QCD. Recently, nonperturbative methods have been developed to treat these theories, based on discrete triangulations of the surfaces that can be generated by simple matrix models. Exact solutions with rich mathematical structure have emerged.

The school was fortunate to have a group of excellent lecturers who are in the forefront of this field. Edouard Brézin introduced large $N$ and matrix model techniques. Sasha Migdal presented the methods of discrete triangulations, discussed the double scaling solution and presented some numerical results on two dimensional geometry. François David gave a series of lectures on the applications of random surface theory to the statistical mechanics of real membranes, as well as some nonperturbative aspects of the matrix model solutions. Sasha Polyakov lectured on the continuum approach to two dimensional quantum gravity. Robbert Dijkgraaf spoke on the topological field theory approach to the subject and I lectured on the $c=1$ matrix models.

This school took place at a very difficult time, during a lull in the Gulf crisis. I would like to thank all the lecturers and participants for braving the clouds of war. We gratefully acknowledge the generous support of the US-Israel Binational Science Foundation, the Rothchild Foundation and the Israeli Ministry of Science. Finally, I thank Steve Weinberg for making the school possible and Tzvi Piran and the local organizing committee for making it a reality.

June 1991

David J. Gross Princeton, New Jersey 


\section{This page is intentionally left blank}




\section{CONTENTS}

Preface

\section{LARGE $N$ LIMIT AND DISCRETIZED TWO-DIMENSIONAL} QUANTUM GRAVITY Edouard Brézin

1. A Review of Large $N$-Techniques in Statistical Mechanics and Field Theory . . . . . . . . . . . . . . . . . . . . . 2 1.1. $N$-Vector Models . . . . . . . . . . . . . . . . 2

1.1.1. The statistical mechanics ' background . . . . . . . 2

1.1.2. Field theory of $N$-vector models . . . . . . . . . . 5

1.1.3. Large $N$ limit of the Schwinger-Dyson equations . . . 6

1.1.4. Large orders of the $1 / N$-expansion . . . . . . . . . 7

1.1.5. The collective field formalism . . . . . . . . . . . 9

1.2. Matrix Models . . . . . . . . . . . . . . . . . . . . 10

2. Matrix Models and Graph Counting . . . . . . . . . . . . . 12

2.1. Discretized 2D Gravity . . . . . . . . . . . . . . . . 12

2.2. Representation of Pure Gravity by a Matrix Integral . . . 14

2.3. The Planar Limit . . . . . . . . . . . . . . . . . . 15

2.4. The Continuum Limit: The "Double Scaling Limit" . . . 19

3. From One Matrix to Multi-Matrix Models . . . . . . . . . . 23

3.1. Double Scaling Limit for Pure Gravity . . . . . . . . . 23

3.2. The Genus Expansion of Pure Gravity . . . . . . . . . 25

3.3. The Ising Model Coupled to Gravity . . . . . . . . . . 27

3.4. Linear Chain of Matrices . . . . . . . . . . . . . . . . . 29

4. Miscellaneous Remarks . . . . . . . . . . . . . . . . . . . 33

4.1. Virasoro Constraints and Loop Equations . . . . . . . . 33

4.2. Unoriented Surfaces . . . . . . . . . . . . . . . . . 35 
Appendix: An Elementary Derivation of the Formula (3.39-3.40) . . 37

References . . . . . . . . . . . . . . . . . . . 38

QUANTUM GRAVITY AS DYNAMICAL TRIANGULATION . . . 41 A. A. Migdal

1. Matrix models and KdV Hierarchy . . . . . . . . . . . . . 41

1.1. Orthogonal polynomials as wave functions . . . . . . . . 41

1.2. Analogy with rotator . . . . . . . . . . . . . . . . . 43

1.3. Double scaling limit and KdV hierarchy . . . . . . . . . 45

1.4. Nonperturbative ambiguities . . . . . . . . . . . . . . 47

2. Stabilization of Matrix Model of Pure Gravity . . . . . . . . 49

2.1. Matrix model as ground state problem . . . . . . . . . 49

2.2. Pure gravity and Gel'fand-Dikiï equation . . . . . . . . 51

2.3. Numerical study . . . . . . . . . . . . . . . . . . . 52

2.4. Summary . . . . . . . . . . . . . . . . . . . 53

3. Simulations of Quantum Gravity . . . . . . . . . . . . . 54

3.1. Introduction . . . . . . . . . . . . . . . . . . . . . 54

3.2. Parallel Monte-Carlo algorithm . . . . . . . . . . . . 56

3.3. Direct simulation of random graphs . . . . . . . . . . . 57

3.4. Numerical results . . . . . . . . . . . . . . . . . . . 60

3.4.1. Mean square extent . . . . . . . . . . . . . . 60

3.4.2. Internal geometry . . . . . . . . . . . . . . . 61

3.4.3. Curvature related characteristics . . . . . . . . . 63

3.4.4. Checking the free field model . . . . . . . . . . 65

3.5. Summary and discussion . . . . . . . . . . . . . . . 69

3.6. 3D gravity as dynamical triangulation . . . . . . . . . 71

3.6.1. Thermodynamics of simplicial gravity . . . . . . 72

3.6.2. Numerical results . . . . . . . . . . . . . . . 74

3.6.3. Summary . . . . . . . . . . . . . . . . . . . 76

References . . . . . . . . . . . . . . . . 76 
INTRODUCTION TO THE STATISTICAL MECHANICS OF RANDOM SURFACES AND MEMBRANES . . . . . . . . . . . 80 Francois David

1. Introduction . . . . . . . . . . . . . . . . . 81

2. Membranes: Physical Systems and Physical Problems . . . . . 82

2.1. Some examples of $2 \mathrm{D}$ flexible objects . . . . . . . . . . 82

2.2. The curvature energy and the Canham-Helfrich model . . 87

2.3. Fluctuations and thermodynamics . . . . . . . . . . . 90

3. Fluid Membranes . . . . . . . . . . . . . . . . . . . . . 93

3.1. Rigidity and tension renormalization by thermal fluctuations 93

3.2. Non-perturbative large $d$ results . . . . . . . . . . . . 97

3.3. Some experimental consequences . . . . . . . . . . . . 99

3.4. On the nature of the "bicontinuous phase" . . . . . . . 102

4. Crystalline Membranes and the Crumpling Transition . . . . . 104

4.1. Classical elasticity theory . . . . . . . . . . . . . 105

4.2. Models for elastic membranes . . . . . . . . . . . . . 106

4.3. Theoretical status of the crumpling transition . . . . . . 109

4.4. Experimental status . . . . . . . . . . . . . . . . . 110

5. Defects and Hexatic Membranes . . . . . . . . . . . . . 111

5.1. Fusion in 2 dimensions . . . . . . . . . . . . . . . . 111

5.2. Model for hexatic membranes . . . . . . . . . . . . . 113

5.3. Renormalization of hexatic membranes . . . . . . . . . 114

5.4. Crumpling of hexatic membranes . . . . . . . . . . . . 115

5.5. Disclinations and melting of the hexatic phase . . . . . . 116

5.6. Numerical and experimental relevance . . . . . . . . . 118

6. Conclusion . . . . . . . . . . . . . . . . . . . 118

References . . . . . . . . . . . . . . . . . 120 
NON-PERTURBATIVE EFFECTS IN TWO DIMENSIONAL QUANTUM GRAVITY

Francois David

1. Introduction . . . . . . . . . . . . . . . . . . . . . 125

2. The Scaling Limits for Pure Gravity . . . . . . . . . . . . . 125

3. The Properties of the Painlevé I Equation . . . . . . . . . . 128

4. Loop Equations . . . . . . . . . . . . . . . . . . . . . . 131

5. Non-Perturbative Effects in Matrix Models . . . . . . . . . . 134

6. Stochastic Quantization and the SUSY 1D String . . . . . . 137

7. Conclusion . . . . . . . . . . . . . . . . . . . . . . . . 139

References . . . . . . . . . . . . . . . . 140

THE $c=1$ MATRIX MODELS . . . . . . . . . . . . 143 David J. Gross

1. Introduction . . . . . . . . . . . . . . . . . . 143

2. Formation of the $c=1$ Matrix Models . . . . . . . . . . 145

3. Reduction to Fermionic Eigenvalues . . . . . . . . . . . . . 147

4. The Classical Solution . . . . . . . . . . . . . . . . . 150

5. The Complete Quantum Solution . . . . . . . . . . . . . 152

6. Solution of the Discrete String . . . . . . . . . . . . . . . 154

7. The Solution on a Circle . . . . . . . . . . . . . 155

8. Non Singlets States and Vortices . . . . . . . . . . . . . . 157

9. Fermionic Field Theory . . . . . . . . . . . . . . . . . . 161 
10. Correlation Functions . . . . . . . . . . . . . . . . . . . 164

11. The Two-Point Functions on the Sphere . . . . . . . . . . . 167

12. Conclusions . . . . . . . . . . . . . . . . . . . 172

References .. . . . . . . . . . . . . . . . 172

SINGULAR STATES IN 2D QUANTUM GRAVITY . . . . . . . 175 Alexander Polyakov

Introduction . . . . . . . . . . . . . . . . . . . . . 175

1. The Models with $C=1$. . . . . . . . . . . . . . . . 175

2. The S-Matrix and Kinematics . . . . . . . . . . . . . . . 177

3. Algebra of Discrete States . . . . . . . . . . . . . . 185

4. Perspectives . . . . . . . . . . . . . . . . . . . . . . . 189

References . . . . . . . . . . . . . . . . . . . . 189

TOPOLOGICAL FIELD THEORY AND 2D QUANTUM GRAVITY . 191 Robbert Dijkgraaf

1. Introduction . . . . . . . . . . . . . . . . . . . 191

2. Topological Field Theory . . . . . . . . . . . . . . . . . . 192

2.1. Topological invariance . . . . . . . . . . . . . . . . 192

2.2. The descent equation . . . . . . . . . . . . . . . . . 195

2.3. Supersymmetry, twisting, and Donaldson theory . . . . . 197

2.4. Ground states and physical states . . . . . . . . . . . 198

2.5. The operator algebra and factorization . . . . . . . . . . 199

2.6. Topological field theory in two dimensions . . . . . . . . 201

2.7. Non-local operators . . . . . . . . . . . . . . . . . . 205

3. Topological Conformal Field Theory . . . . . . . . . . . . . 206 
3.1. Twisting $N=2$ superformal models . . . . . . . . . . 207

3.2. Generalized $\operatorname{SL}(2, \mathrm{C})$ invariance and its consequences . . . 210

3.3. Examples of topological CFT's . . . . . . . . . . . . . 211

3.4. Critical string theory . . . . . . . . . . . . . . . . . 213

4. Topological String Theory

4.1. Action and symmetries . . . . . . . . . . . . . . . . 216

4.2. Pure topological gravity . . . . . . . . . . . . . . . . 218

4.3. Contact and factorization terms . . . . . . . . . . . . 219

5. Matrix Models and the KdV Hierarchy . . . . . . . . . . . . 223

5.1. Multi-critical prints . . . . . . . . . . . . . . . . . 223

5.2. The Virasoro algebra . . . . . . . . . . . . . . . . . 226

5.3. Loop equations . . . . . . . . . . . . . . . . . . . 227

5.4. The KdV hierarchy . . . . . . . . . . . . . . . . . . 229

5.5. Multimatrix models . . . . . . . . . . . . . . . . . 230

5.6. Twisted $N=2$ minimal models . . . . . . . . . . . . 232

5.7. $W$-constraints . . . . . . . . . . . . . . . . . 236

References . . . . . . . . . . . . . . . 237

List of Participants . . . . . . . . . . . . . . . . . . . . . . 239 


\section{TWO DIMENSIONAL QUANTUM GRAVITY AND RANDOM SURFACES}

